\title{
La difusión de la producción científica en el ámbito de las Humanidades: el caso de la Universidad de León
}

\author{
Andrés Fernández-Ramos* \\ Leticia Barrionuevo**
}

Artículo recibido:

17 de junio de 2021

Artículo aceptado:

15 de septiembre de 2021

Artículo de investigación

\section{Resumen}

La difusión de la producción científica se ha convertido en un nuevo quehacer de los investigadores. Además de los tradicionales, han surgido otros canales para dar a conocer esas publicaciones al resto de la comunidad científica, como las redes sociales académicas, los identificadores de autores o los repositorios institucionales. Se compara el uso que hacen los investigadores del área de Humanidades de la Universidad de León de cinco plataformas de difusión de producción científica: ORCID, Google Scholar, ResearchGate, Academia.edu, y el repositorio institucional de la Universidad de León. Se analiza la presencia de los

* Área de Biblioteconomía y Documentación, Universidad de León, España afernr@unileon.es

** Facultad de Filosofía y Letras, Universidad de León, España buffl@unileon.es 
investigadores en estas plataformas y la producción científica depositada o vinculada a su perfil. Se observa una gran presencia de estos investigadores en ORCID y en el repositorio institucional, aunque en muchos casos con perfiles incompletos y desactualizados. La presencia en redes sociales académicas es menor y los perfiles creados tampoco recogen toda la producción científica de sus autores; sin embargo, para depositar copias en acceso abierto, los investigadores prefieren las redes sociales al repositorio institucional. Es necesario fomentar el uso de las plataformas de difusión de producción científica, especialmente de los repositorios institucionales, siendo la biblioteca un agente fundamental en esta labor.

Palabras clave: Redes Sociales Académicas; Repositorios Institucionales; Humanidades; Difusión de la Investigación

The dissemination of scholarly publications in $\mathrm{Hu}-$ manities: The case of the University of León Andrés Fernández-Ramos and Leticia Barrionuevo

\section{Abstract}

The dissemination of scholarly publications has become a new task for researchers. In addition to the traditional channels, other new ones have emerged to make this kind of publications known to the rest of the scientific community, such as academic social networks, author identifiers or institutional repositories. In this article, it is compared the use made by researchers in the Humanities area of the University of León of five platforms for the dissemination of scientific production: ORCID, Google Scholar, ResearchGate, Academia.edu and the institutional repository of the University of León. The presence of researchers on these platforms and the scientific production deposited or linked to their profile are analyzed. A large presence of these researchers is observed in ORCID and in the institutional repository, although, in many cases, their profiles are incomplete and outdated. Their presence in academic social networks is lower and the profiles created do not include all the scientific production of their authors. However, to deposit copies in open access, researchers prefer social networks 
to institutional repositories. It is necessary to promote the use of platforms for the dissemination of scientific production, especially institutional repositories, with the library being a key agent in this task.

Keywords: Academic Social Networks; Humanities; Institutional Repositories; Research Dissemination

\section{INTRODUCCIÓN}

Z $\mathrm{n}$ el mundo académico es habitual que los investigadores apoyen el acce工so abierto en todas sus modalidades como estrategia para promocionar, difundir y dar visibilidad a sus trabajos, comprendiendo las ventajas que este modelo supone para impulsar la comunicación de la ciencia (Ruiz-Pérez y Delgado-López-Cózar, 2017; Rodríguez Bravo y Nicholas, 2020). Aunque parece que el modelo de publicación en acceso abierto es inevitable a largo plazo, su implantación en el mundo científico ha sido muy lento debido, principalmente, al patrón de oligopolio que tiene la industria editorial (Björk, 2017). Cada vez hay más contenidos científicos en acceso abierto, pero el porcentaje de artículos científicos depositados en repositorios o publicados en revistas Open Access aún es escaso (Boock et al., 2020).

El desarrollo de la web social ha favorecido cambios significativos en los entornos de comunicación científica, ya que ahora es más fácil compartir investigación y recursos a través de plataformas abiertas, como los blogs, portales académicos, redes sociales o webs especializadas, entre otros. Los investigadores, cada vez más conscientes de la importancia de tener visibilidad e impacto en la web, han visto las ventajas de adoptar un papel proactivo en la difusión de su trabajo en la red y en la creación de su propia identidad digital, definida por Fernández-Marcial y González-Solar (2015: 657) como "el resultado del esfuerzo consciente que realiza el investigador por y para ser identificado y reconocido en un contexto digital, distinguiéndose del conjunto de investigadores a través de la normalización, con el uso de identificadores, y la difusión de resultados de investigación en redes y plataformas de diversa naturaleza". Los identificadores, los perfiles de autor, los repositorios y las redes sociales académicas (RSA) son las herramientas más utilizadas en este sentido. Todas ellas aportan servicios y ventajas para potenciar la identidad digital, pero también requieren un considerable esfuerzo para mantenerlas actualizadas y sacarles provecho (García-Peñalvo, 2018).

Los identificadores y perfiles de autor permiten la identificación unívoca de los investigadores a partir de la asignación de un código alfanumérico que 
los diferencia de otras personas. Son herramientas muy útiles, ya que unifican las diferentes formas del nombre de firma que figuran en sus trabajos y vinculan las publicaciones a los autores. Además, los sitios web de estas herramientas permiten añadir información de contacto, sobre su formación, líneas de investigación u otros aspectos relevantes de su CV, de forma que sirven de carta de presentación del investigador en la web. Uno de los más conocidos es el ORCID, que es el estándar que se ha implantado en los últimos años como identificador principal por su carácter abierto, centrado en el usuario y por su interoperabilidad con otros sistemas análogos (Marín-Arraiza y Mejías, 2020).

Los repositorios institucionales, por su parte, se concibieron desde su origen como servicios vivos, útiles para la gestión, difusión y preservación de las publicaciones de los investigadores, con el objetivo de ampliar su uso e impacto (Lynch y Lippincott, 2005; McDowell, 2007). Su papel es especialmente relevante en el ecosistema del acceso abierto, ya que son plataformas específicamente orientadas a ese fin. Los primeros repositorios que se implementaron fueron poco amigables y difíciles de manejar, pero con el tiempo han ido mejorando, incorporando arquitecturas más amigables, que se integran con las estructuras académicas globales existentes. Son repositorios de nueva generación, que permiten el depósito persistente de objetos digitales, tales como documentos, datos y códigos (Becerril García y Aguado López, 2020), y que incluyen servicios de valor añadido, como los ORCID de los autores, métricas de los documentos, información sobre derechos de autor o la posibilidad de descargar registros (Baessa et al., 2015; Scherer, 2016; Fernández-Ramos y Barrionuevo, 2021).

Las RSA, como ResearchGate y Academia.edu, son plataformas que proporcionan un espacio idóneo para que los investigadores se comuniquen, construyan redes profesionales y difundan y compartan sus publicaciones (Thelwall y Kousha, 2015; Xia et al., 2016; Martín-Martín et al., 2017). Sus principales usos son la búsqueda y obtención de documentos, la formación de una identidad digital (Meishar-Tal y Pieterse, 2017), la difusión de resultados y actividades de investigación (Nández y Borrego, 2013; Campos-Freire y Rúas-Araújo, 2016) o la medición del impacto académico a través de las métricas adicionales que ofrecen (Gruzd, Staves y Wilk, 2012; Ovadia, 2013).

Todos estos son recursos valiosos para que los investigadores creen una identidad digital y difundan y den visibilidad a su producción científica, sobre todo en el caso de las tesis, actas de congresos, informes u otro tipo de documentos que cuentan con menos presencia en la mayoría de las bases de datos académicas. Sin embargo, su uso por parte de los investigadores es bastante desigual entre la comunidad académica. Por un lado, ha aumentado el 
número de investigadores con ORCID (Martínez-Méndez y López-Carreño, 2019) y con perfiles académicos en plataformas como Google Scholar (Ortega, 2015a). Por otra parte, con respecto al depósito en acceso abierto de la producción científica, los repositorios institucionales no son muy usados por los investigadores para dar visibilidad a sus publicaciones, a menos que sea una exigencia o un mandato por la entidad financiadora que subvenciona la investigación (Borrego, 2016; Fathli, Lundén y Sjögårde, 2014). Por el contrario, las RSA se han posicionado como la principal opción de los investigadores para dar difusión a sus trabajos, debido principalmente a su facilidad de uso, mejores prestaciones, rapidez en la difusión de información o la inclusión de multitud de servicios de valor añadido, entre otras ventajas (Davis y Connolly, 2007; Borrego, 2017). En el fondo, repositorios y RSA no son excluyentes, sino complementarios, tal y como señalan Melero Melero (2020) o Gómez-Castaño (2020). Los repositorios institucionales deben incluir la mayor parte de los trabajos producidos por la comunidad de su universidad o centro de investigación y las RSA deben de servir de medio de comunicación entre investigadores, por lo que la unión entre ambas es una buena combinación (Sánchez Pérez, 2020).

\section{OBjetivos y metodología}

El objetivo general de este trabajo es analizar el uso que hacen los investigadores del área de Humanidades de la Universidad de León de cinco plataformas de difusión de producción científica: ORCID, Google Scholar (GS), ResearchGate (RG), Academia.edu y el repositorio institucional de la Universidad de León (BULERIA). Este objetivo general se concreta en los siguientes objetivos específicos: constatar la presencia de los investigadores en estas plataformas, averiguar qué publicaciones aparecen vinculadas a esos perfiles, cuáles son sus características y cuáles de ellas han sido depositadas para su consulta en acceso abierto.

Para alcanzar el objetivo propuesto se siguió una metodología descriptiva basada en el análisis del contenido de las cinco plataformas y articulada en tres etapas: identificación de los investigadores que conforman la población; recogida de datos sobre la presencia de los investigadores en las plataformas, documentos vinculados y documentos depositados en acceso abierto, y análisis de los datos recogidos.

La población está conformada por los investigadores del área de Humanidades de la Universidad de León. Únicamente se incluyó el profesorado a tiempo completo que estuviera en activo a final de 2019, lo que excluyó a 
los profesores asociados (a tiempo parcial) y a los jubilados. Se consideraron pertenecientes al área de Humanidades a los investigadores adscritos a las áreas de conocimiento que la Agencia Nacional de Evaluación de la Calidad y la Acreditación engloba en la categoría "Artes y Humanidades". Todas estas áreas de conocimiento, en el caso de la Universidad de León, están integradas en alguno de los cinco departamentos ubicados en la Facultad de Filosofía y Letras. En el Anexo se puede consultar la distribución de los 96 investigadores pertenecientes a estas 21 áreas y cinco departamentos incluidos en el estudio.

La recogida de datos se realizó en mayo de 2020. Para un acopio normalizado de la información se utilizó una plantilla en la que se consignó la siguiente información:

- Presencia del investigador en la plataforma. Se consideró que un investigador tiene presencia en las plataformas analizadas si tiene un perfil en ellas (aunque no haya publicaciones). En el caso del repositorio institucional, puesto que no es posible crearse un perfil, se consideró que tiene presencia si ha depositado al menos un documento. Para localizar el perfil de cada investigador en las cinco plataformas se buscó por nombre y apellidos, teniendo en cuenta las posibles formas en que pueden aparecer: iniciales, uso de uno o dos apellidos, guiones entre apellidos, etc. Se comprobaron los datos de filiación de cada investigador para asegurarnos de que era la persona buscada.

- Número de documentos en cada perfil. Se contabilizó el número de publicaciones científicas que cada investigador tenía en cada plataforma publicadas entre 2010 y 2018. De esta forma, con más de un año de plazo, se dio tiempo suficiente a los investigadores para que incluyeran esos documentos en sus perfiles. Se eliminaron duplicados en el caso de que un investigador tuviera más de una versión del mismo documento.

- De cada documento se consignó la fecha de publicación del original; la tipología documental, considerándose cinco posibilidades de documento científico: artículo de revista, monografía, capítulo de libro, comunicación a congreso, y otros (tesis, prólogos, reseñas, comentarios), y, en el caso de RG o Academia.edu, si el documento está en acceso abierto.

Los datos fueron analizados de forma descriptiva con el programa Excel de Microsoft, obteniéndose frecuencias y porcentajes y creando gráficos que facilitaran la comprensión de los datos. 


\section{Resultados}

Los resultados obtenidos muestran que en líneas generales los investigadores del área de Humanidades de la Universidad de León hacen uso de las plataformas de difusión de la producción científica y la gran mayoría cuenta con un perfil en varias de ellas. Sólo un investigador no tiene presencia en ninguna de las plataformas, mientras que $26 \%$ tiene presencia en las cinco, 37,5\% en cuatro de ellas, $19,8 \%$ en tres y $15,6 \%$ en dos de ellas.

Como puede apreciarse en la Figura 1, las plataformas con mayor presencia de los investigadores son ORCID y BULERIA, con más de $95 \%$ cada una. El uso de las RSA y de GS es menor, aunque no desdeñable: 68,75 \% de los investigadores tiene perfil creado en Academia.edu, 63,54 \% lo tiene en GS y $45,83 \%$ en RG.

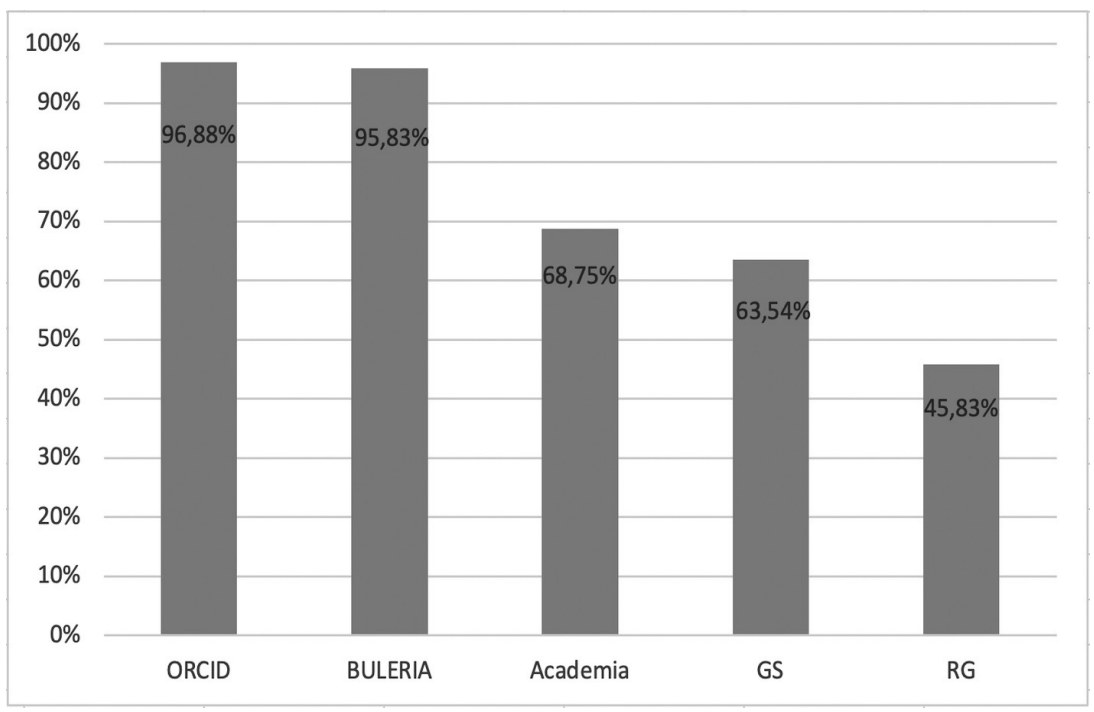

Figura 1. Presencia de los investigadores en las plataformas

\section{Documentos referenciados}

Más allá de la mera presencia o la creación de un perfil, se ha analizado el grado de utilización de las plataformas para difundir su producción científica y se ha podido observar que es muy desigual entre los investigadores incluidos en el estudio. De ellos, 83 (86,5 \%) han introducido datos de sus publicaciones en alguna de las plataformas, mientras que 13 investigadores no lo 
han hecho y únicamente cuentan con un perfil en alguna plataforma, pero sin aportar datos de sus publicaciones. El número medio de publicaciones que los investigadores han referenciado en su perfil es de 25 y la mediana es 21,5 , aunque como se puede apreciar en la Figura 2, existe una gran disparidad entre los documentos que cada investigador ha incluido en su perfil. Más de $30 \%$ de los investigadores (eje de abscisas) ha referenciado menos de 10 documentos publicados (eje de ordenadas) entre 2010 y 2018 en estas plataformas y algo más de $10 \%$ de los investigadores ha mencionado más de 50 .

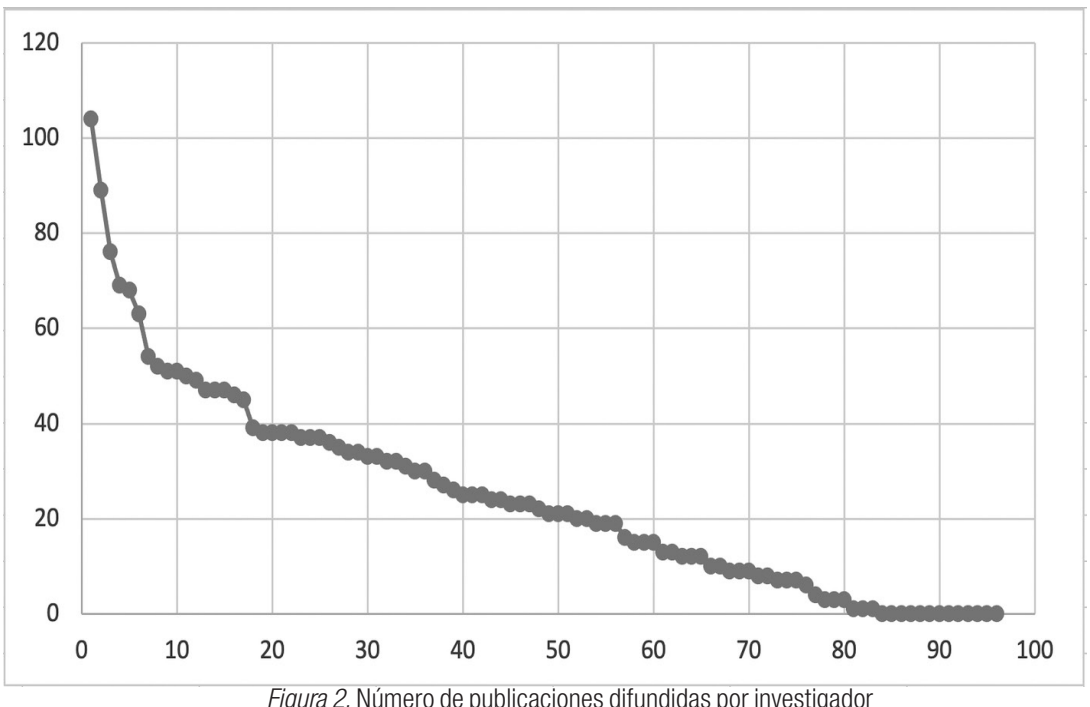

Figura 2. Número de publicaciones difundidas por investigador

El número de publicaciones incluidas en cada perfil también varía en función de la plataforma (Figura 3), lo que muestra las preferencias de los investigadores respecto a dónde difundir la información de su producción científica. De los 2354 documentos diferentes identificados en el conjunto de las cinco plataformas, $68 \%$ aparecen en ORCID, $56 \%$ en GS y, a mucha distancia, $26 \%$ en RG, $17 \%$ en Academia.edu y $11 \%$ en BULERIA. Es destacable el uso de ORCID para difundir la producción científica. Asimismo, llama la atención que, a pesar de que hay más investigadores con perfil en Academia.edu que en RG, haya bastantes más documentos en RG. Una posible explicación sería la mayor facilidad para incorporar los datos de los documentos en RG. Otra explicación sería que algunos investigadores únicamente hayan creado un perfil en Academia.edu para poder acceder al texto completo de los documentos depositados, requisito que no es necesario en RG. 


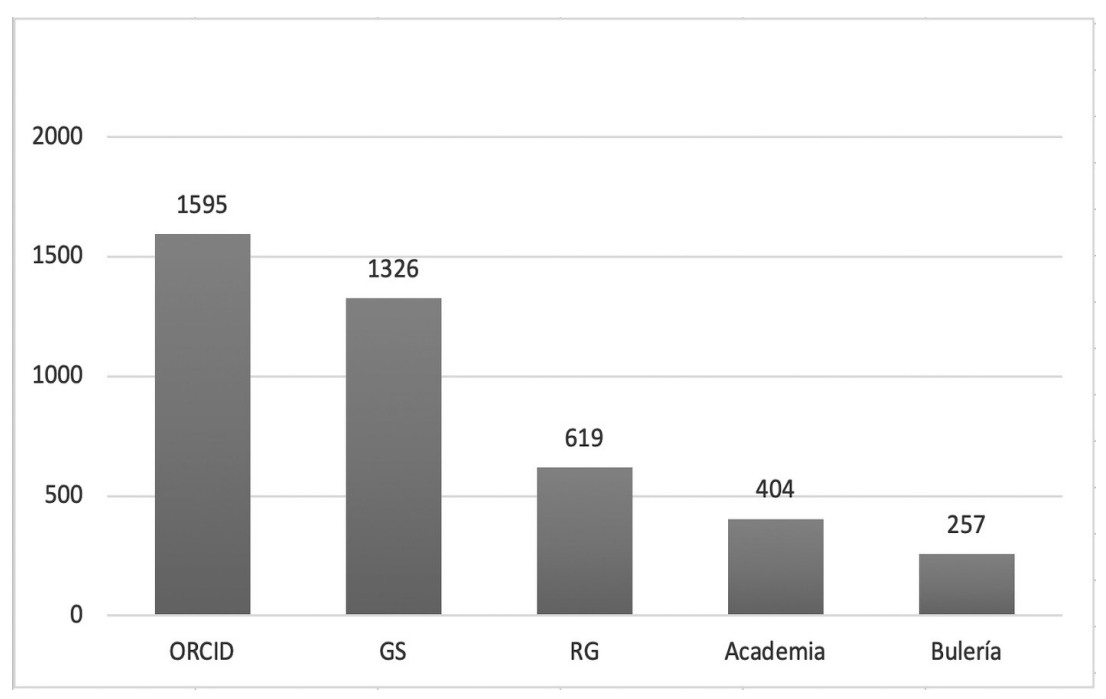

Figura 3. Documentos referenciados en cada plataforma

Al analizar con más profundidad las características de los documentos referenciados en estas cinco plataformas, se ha podido observar que, en líneas generales, predominan los artículos de revistas y los capítulos de libro: más de $40 \%$ del total de los documentos referenciados son artículos y casi $30 \%$ son capítulos, mientras que los libros completos apenas superan $7 \%$, tal y como se puede apreciar en la Figura 4. Existen ciertas diferencias entre plataformas, sin que se pueda hablar de una gran variabilidad. En ORCID, que es donde más documentos hay referenciados, los artículos no tienen tanto protagonismo como en el resto de plataformas. De hecho, hay más capítulos de libro y es también la plataforma con más contribuciones a congresos. En el resto de plataformas, con menos documentos, los investigadores son más selectivos y priorizan la difusión de los artículos sobre otras tipologías documentales. Las dos RSA, RG y Academia.edu, muestran una distribución bastante parecida, pero con más peso de los capítulos de libro en Academia.edu y más aportaciones a congresos en RG. Por otra parte, es destacable que en GS hay un elevado número de capítulos de libro, con casi un tercio de las publicaciones, y que tanto en BULERIA como en RG hay menos capítulos de libro que en el resto de plataformas. 


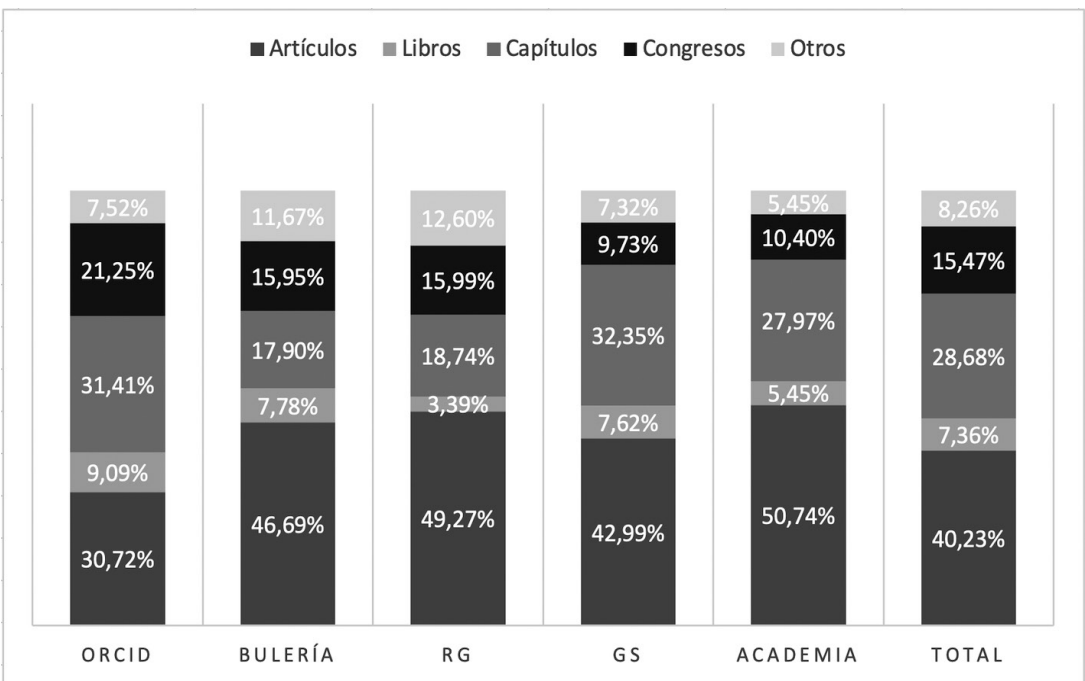

Figura 4. Tipología de las publicaciones referenciadas

Con respecto a la antigüedad de los documentos referenciados, se observa que predominan las publicaciones más antiguas, aquellas publicadas desde 2010 hasta 2014. Los documentos más recientes, publicados desde 2015, tienen menos presencia, como se aprecia en la Figura 5; ello a pesar de que la ventana de análisis en este estudio abarcaba hasta 2018 para poder dar tiempo a los investigadores a que incorporen esa información en las plataformas. Esto nos lleva a pensar que los investigadores no actualizan mucho su información en las plataformas, sobre todo en ORCID, donde hay una menor carga de documentos recientes.

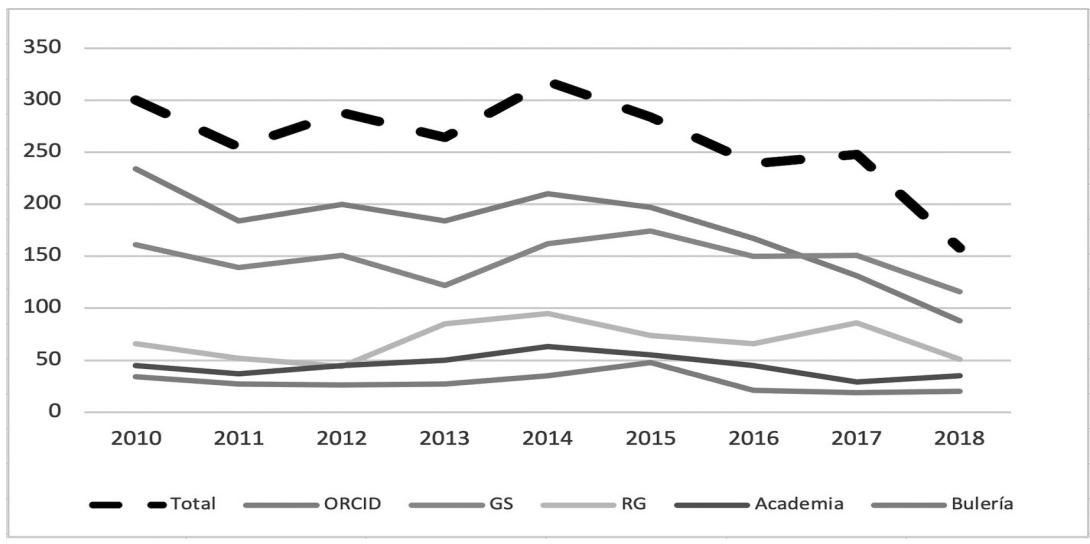

Figura 5. Fecha de publicación de los documentos referenciados 


\section{Documentos depositados en acceso abierto}

En este apartado se desglosan los resultados sobre el depósito en acceso abierto del texto completo de los documentos referenciados en aquellas plataformas que lo permiten, es decir, en RG, Academia.edu y BULERIA. Tanto ORCID como GS permiten identificar y vincular la producción científica de un investigador, pero no el depósito de documentos.

En primer lugar, hay que destacar que 31,82\% de los 2354 documentos que aparecen en alguna de las cinco plataformas analizadas disponen de una copia en acceso abierto en alguna de las tres plataformas que lo permiten. En el caso de BULERIA, al tratarse de un repositorio institucional, $100 \%$ de los 257 documentos referenciados están depositados. En RG, 361 de los 619 documentos referenciados cuentan con una copia en acceso abierto (58,32\%), mientras que en Academia.edu se ha depositado una copia en acceso abierto en 302 de los 404 documentos referenciados (74,75\%). Estos datos también demuestran que el porcentaje de documentos depositados en las RSA es mayor que el de los repositorios institucionales.

Uno de los resultados más sorprendentes con respecto al depósito de documentos es el escaso grado de solapamiento entre plataformas: los investigadores no depositan los mismos documentos en todas las plataformas, sino que hay una gran disparidad con respecto a lo que suben en unas y en otras. Así, como puede observarse en la Figura 6, únicamente 17 publicaciones han sido depositadas en acceso abierto en las tres plataformas $(2,3 \%$ de las publicaciones depositadas). En esta figura se observa que la gran mayoría de las publicaciones únicamente se ha depositado en una de las tres plataformas, existiendo un solapamiento muy reducido entre pares de plataformas.

Con respecto a la tipología de los documentos depositados, se aprecia una alta y lógica correspondencia con la tipología de los documentos referenciados (Figura 7). Se ha observado un notable predominio de los artículos de revista sobre otros tipos documentales, así como una escasa presencia de libros en las tres plataformas, sobre todo en las RSA. En Academia.edu existe una mayor proporción de capítulos de libro que en RG y BULERIA, mientras que las aportaciones a congresos u otras tipologías documentales tienen un nivel de depósito considerablemente menor. 


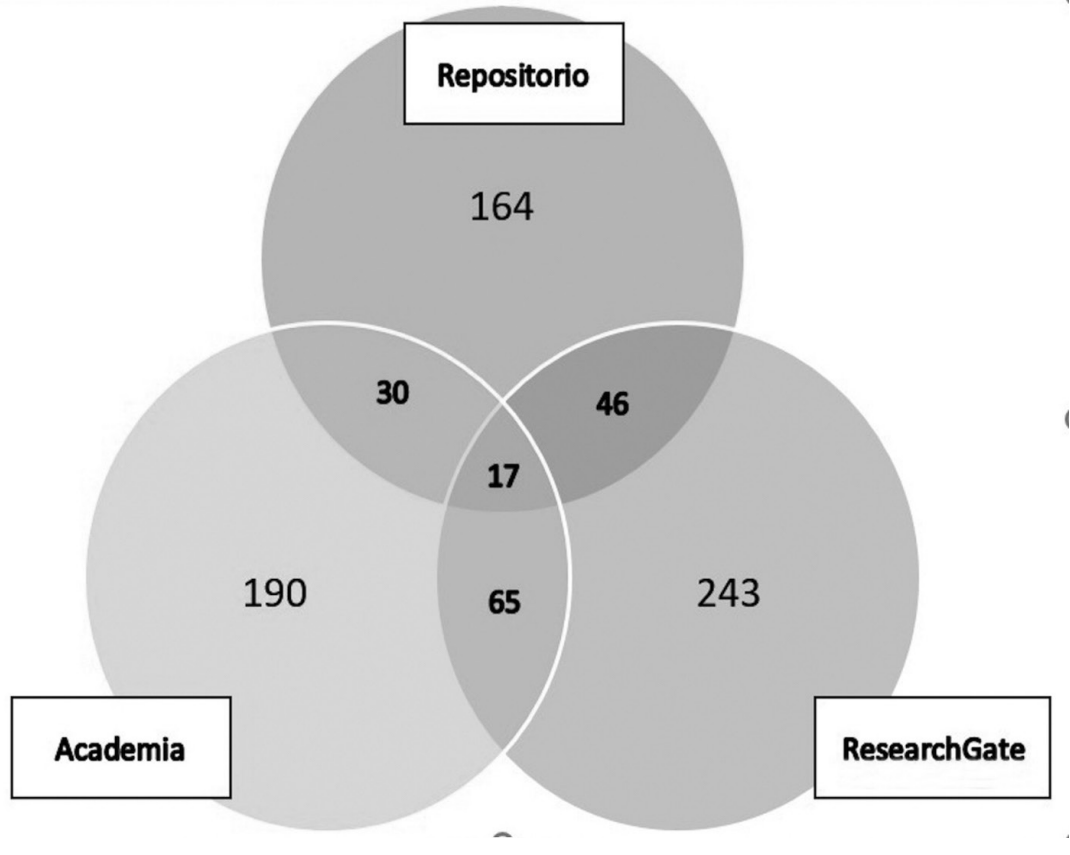

Figura 6. Solapamiento de documentos depositados entre plataformas

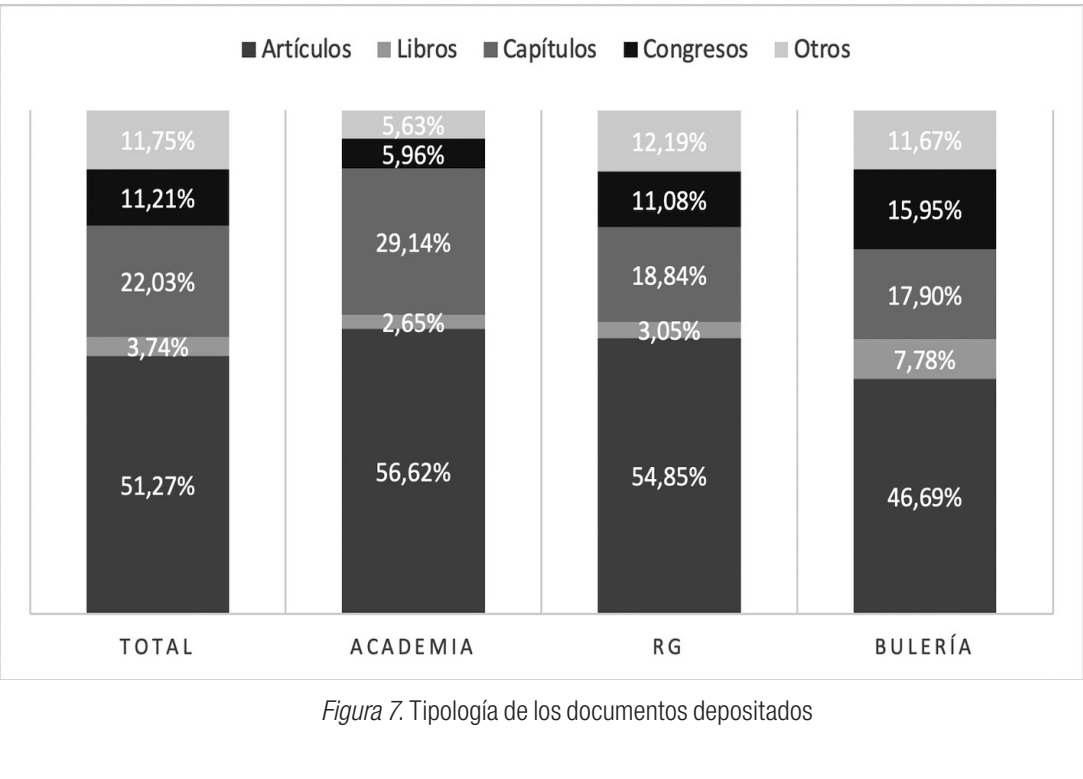




\section{DisCUSIÓN Y CONCLUSIONES}

Los resultados de este trabajo ofrecen un panorama del uso que hacen los investigadores del área de Humanidades de la Universidad de León de cinco de las principales plataformas de difusión de producción científica y están en clara consonancia con la literatura científica publicada sobre el tema. Así, se ha podido comprobar que, en líneas generales, los investigadores incluidos en el estudio conocen y utilizan estas plataformas, ya que sólo un investigador no tiene presencia en ninguna de los servicios analizados y $26 \%$ la tiene en las cinco. El uso generalizado de estas plataformas ya ha sido puesto de manifiesto en otros trabajos, como en el de Mikki et al. (2015) sobre la presencia digital de los investigadores noruegos en ResearchGate, Academia.edu, Google Scholar, ResearchID y ORCID, o en el de Prieto Gutiérrez (2018), centrado en autores del área de Biblioteconomía y Documentación de universidades españolas.

Otro resultado relevante obtenido en esta investigación ha sido que la plataforma más empleada es ORCID, en la que tienen creado un perfil $96 \%$ de los investigadores incluidos en el estudio. Este uso cada vez mayor del ORCID ya ha sido puesto de manifiesto en el trabajo de Marín-Arraiza y Mejías (2020), donde se expone que es el estándar que se ha implantado en los últimos años como identificador principal, bien por su carácter abierto, centrado en el usuario y por su interoperabilidad con otros sistemas análogos. Este identificador, además, está cobrando especial importancia, ya que está siendo requerido en convocatorias de proyectos de investigación y es usado en muchas revistas y bases de datos para identificar unívocamente a los autores (Martínez-Méndez y López-Carreño, 2019).

Los resultados muestran también que la mayoría de los investigadores analizados cuenta con perfil en alguna RSA: más de dos tercios en Academia.edu y casi la mitad en ResearchGate. Esta buena aceptación de las RSA en el ámbito de las Humanidades ya había sido detectada en otros trabajos, como el de Nández y Borrego (2013) o el de Al-Aufi y Fulton (2014), y la preferencia en esta área por Academia.edu con respecto a ResearchGate también había sido observado en otros estudios. Así, Mikki et al. (2015) muestran que en todas las disciplinas impartidas en la Universidad de Bergen ResearchGate es la plataforma más utilizada por los investigadores del ámbito de las ciencias, mientras que Academia.edu es la preferida en la Facultad de Humanidades. Yan et al. (2021) indican la limitada presencia en ResearchGate de los investigadores de Artes y Humanidades en las universidades de Estados Unidos. En España, Ortega (2015b) también mostró cómo los investigadores de Humanidades y Ciencias Sociales del CSIC eran los que más utilizaban Academia.edu mientras que en el resto de disciplinas se usaba más ResearchGate. 
El menor uso observado del repositorio en relación con las RSA en esta investigación también coincide con otros trabajos previos (Borrego, 2017; Eva y Wiebe, 2019; Mason, 2020; Orduña-Malea, 2020). Esta circunstancia debería ser motivo de preocupación para los responsables de repositorios institucionales, que ven cómo su esfuerzo por poner a disposición de sus investigadores servicios de preservación y difusión de la producción científica son infrautilizados, pero también podría servir para observar y aprender de las RSA e incorporar a los repositorios aquellos elementos que pudieran facilitar su uso y adaptarse a las necesidades y preferencias de los investigadores (Fernández-Ramos y Barrionuevo, 2021).

Los datos descriptivos que hemos obtenido en este trabajo sirven de "diagnóstico" del uso de las plataformas académicas por un grupo de investigadores de Humanidades, pero para tener un mayor conocimiento del uso y del papel y relevancia de estas plataformas es necesario realizar más investigaciones en otros ámbitos temáticos y en otras instituciones, hacer comparaciones y ver su evolución temporal. Asimismo, este tipo de análisis debe ser complementado con estudios cualitativos que permitan conocer las motivaciones de los investigadores para usar o no usar estas plataformas, así como las barreras y alicientes que perciben en cada una de ellas.

La difusión de la producción científica a través de las distintas plataformas académicas redunda en más visibilidad e impacto de las publicaciones, y por tanto en una mayor visibilidad de los investigadores y de las instituciones en las que trabajan. En este sentido, consideramos que es necesario elaborar estrategias institucionales que apoyen a los investigadores en la creación y alimentación de sus perfiles de autores, así como en el depósito en acceso abierto de su producción científica en repositorios institucionales y en RSA. En el caso de la Universidad de León, la biblioteca se ha implicado en esta labor y ha apoyado e los investigadores, ayudándoles a crear y alimentar el ORCID y los perfiles de GS. También ayuda a los investigadores a depositar sus documentos en el repositorio institucional a través del archivo delegado $\mathrm{y}$, con respecto a las RSA, se han impartido cursos de formación sobre el uso de RG y Academia.edu, pero en este caso han sido los propios investigadores quienes se han encargado de gestionar sus perfiles.

Consideramos que las bibliotecas universitarias y centros de investigación pueden y deben jugar un papel relevante en la promoción y formación de sus investigadores con respecto a estrategias para mejorar la difusión, visibilidad e impacto de su producción científica, ya que beneficia tanto a ellos como a sus instituciones. Esto es especialmente importante en el caso del uso del repositorio institucional, donde la implicación de la biblioteca debe ser aún mayor ya que la gestiona la propia institución, al contrario de las otras 
plataformas, que son externas. Los bibliotecarios deben ofrecer apoyo a los investigadores para que estos puedan cumplir con los mandatos relativos al acceso abierto y ayudarles en el depósito de documentos, ya que saben qué versiones alojar en los repositorios sin infringir los derechos de autor, cómo deben cumplimentarse los formularios con los metadatos, etc. No obstante, también es necesario impulsar la creación de repositorios más eficaces, con funcionalidades más avanzadas y servicios de valor añadido que animen a los investigadores a utilizarlos.

\section{REFERENCIAS}

Al-Aufi, Ali S. y Crystal Fulton. 2014. "Use of Social Networking Tools for Informal Scholarly Communication in Humanities and Social Sciences Disciplines". Procedia - Social and Behavioral Sciences 147: 436-445.

https://doi.org/10.1016/j.sbspro.2014.07.135

Baessa, Mohamed, Thibaut Lery, Daryl Grenz y J. K. Vijayakumar. 2015. "Connecting the Pieces: Using ORCIDs to Improve Research Impact and Repositories”. F1000Research 4. https://doi.org/10.12688/f1000research.6502.1

Becerril García, Arianna y Eduardo Aguado López. 2020. “Una infraestructura abierta distribuida para la comunicación y evaluación científica: el reto para los repositorios institucionales". Revista PH 100: 124-126. https://doi.org/10.33349/2020.100.4669

Björk, Bo Christer. 2017. "Open Access to Scientific Articles: A Review of Benefits and Challenges". Internal and Emergency Medicine 12 (2): 247-253. https://doi.org/10.1007/s11739-017-1603-2

Boock, Michael, Tania Yordanova Todorova, Tereza Stoyanova Trencheva y Radostina Todorova. 2020. "Bulgarian Authors' Open Access Awareness and Preferences”. Library Management 41 (2/3): 91-102. https://doi.org/10.1108/LM-08-2019-0059

Borrego, Ángel. 2016. "Measuring Compliance with a Spanish Government Open Access Mandate". Journal of the Association for Information Science and Technology 67 (4): 757-764. https://doi.org/10.1002/asi.23422

Borrego, Ángel. 2017. "Institutional Repositories versus ResearchGate: The Depositing Habits of Spanish Researchers”. Learned Publishing 30 (3): 185-192. https://doi.org/10.1002/leap.1099

Campos-Freire, Francisco y Raúl Rúas-Araújo. 2016. "Uso de las redes sociales digitales profesionales y científicas: El caso de las tres universidades gallegas". El profesional de la información 25 (3): 431-440. https://doi.org/10.3145/epi.2016.may.13

Davis, Philip M. y Matthew J. L. Connolly. 2007. "Institutional Repositories: Evaluating the Reasons for Non-Use of Cornell University's Installation of DSpace”. DLib Magazine 13 (3-4). https://doi.org/10.1045/march2007-davis 
Eva, Nicole C. y Tara A. Wiebe. 2019. "Whose Research Is It Anyway? Academic Social Networks Versus Institutional Repositories". Journal of Librarianship and Scholarly Communication 7 (1). https://doi.org/10.7710/2162-3309.2243

Fathli, Margareta, Tomas Lundén y Peter Sjögårde. 2014. "The Share of Open Access in Sweden 2011 - analyzing the OA outcome from Swedish universities". ScieCom Info 10 (2). https://journals.lub.lu.se/sciecominfo/article/view/11645

Fernández-Marcial, Viviana y Llarina González-Solar. 2015. "Promoción de la investigación e identidad digital: el caso de a Universidade da Coruña”. El profesional de la información 24 (5): 656-664. https://doi.org/10.3145/epi.2015.sep.14

Fernández-Ramos, Andrés y Leticia Barrionuevo. 2021. "Value-Added Services in Institutional Repositories in Spanish Public Universities”. Information Research 26 (1). https://doi.org/10.47989/IRPAPER895

García-Peñalvo, Francisco José. 2018. "Identidad digital como investigadores. La evidencia y la transparencia de la producción científica". Education in the Knowledge Society 19 (2): 7-28. https://doi.org/10.14201/eks2018192728

Gómez-Castaño, Javier. 2020. "Repositorios digitales y redes sociales académicas: ¿una coexistencia necesaria?” Revista PH 100: 73-75. https://doi.org/10.33349/2020.100.4628

Gruzd, Anatoliy, Kathleen Staves y Amanda Wilk. 2012. "Connected Scholars: Examining the Role of Social Media in Research Practices of Faculty Using the UTAUT Model". Computers in Human Behavior 28 (6): 2340-2350. https://doi.org/10.1016/j.chb.2012.07.004

Lynch, Clifford A. y Joan K. Lippincott. 2005. "Institutional Repository Deployment in the United States as of Early 2005”. D-Lib Magazine 11 (9). https://doi.org/10.1045/september2005-lynch

Marín-Arraiza, Paloma y Gabriela Mejías. 2020. "Identificadores persistentes: la adopción del ORCID ID en España”. Anuario ThinkEPI 14 (1). https://dialnet.unirioja.es/servlet/articulo?codigo $=7541844$ \&orden $=0$ \&info=link

Martín-Martín, Alberto, Enrique Orduna-Malea, Anne Wil Harzing y Emilio Delgado López-Cózar. 2017. "Can We Use Google Scholar to Identify Highly-Cited Documents?" Journal of Informetrics 11 (1): 152-163. https://doi.org/10.1016/j.joi.2016.11.008

Martínez-Méndez, Francisco Javier y Rosana López-Carreño. 2019. “La paulatina adopción de ORCID para la mejora de la identidad digital de las revistas científicas españolas en acceso abierto”. Investigación Bibliotecológica 33 (80): 73-95. https://doi.org/10.22201/iibi.24488321xe.2019.80.57994

Mason, Shannon. 2020. "Adoption and Usage of Academic Social Networks: A Japan Case Study”. Scientometrics 122 (3): 1751-1767. https://doi.org/10.1007/s11192-020-03345-4

McDowell, Cat S. 2007. "Evaluating Institutional Repository Deployment in American Academe since Early 2005: Repositories by the Numbers, Part 2”. D-Lib Magazine 13 (9-10). https://doi.org/10.1045/september2007-mcdowell 
Meishar-Tal, Hagit y Efrat Pieterse. 2017. "Why Do Academics Use Academic Social Networking Sites?" International Review of Research in Open and Distributed Learning 18 (1). https://doi.org/10.19173/irrodl.v18i1.2643

Melero Melero, Remedios. 2020. "Los porqués de los repositorios de acceso abierto y las redes académicas”. Revista PH 100: 60-65. https://doi.org/10.33349/2020.100.4690

Mikki, Susanne, Marta Zygmuntowska, Yvind Liland Gjesdal y Hemed Ali Al Ruwehy. 2015. "Digital Presence of Norwegian Scholars on Academic Network Sites-Where and Who Are They?” PLoS ONE 10 (11): e0142709. https://doi.org/10.1371/journal.pone.0142709

Nández, Gemma y Ángel Borrego. 2013. "Use of Social Networks for Academic Purposes: A Case Study”. Electronic Library 31 (6): 781-791. https://doi.org/10.1108/EL-03-2012-0031

Orduña-Malea, Enrique. 2020. "De la aldea pública de los repositorios institucionales a la metrópoli privada de ResearchGate”. Revista PH 100: 102-104. https://doi.org/10.33349/2020.100.4659

Ortega, José Luis. 2015a. "How Is an Academic Social Site Populated? A Demographic Study of Google Scholar Citations Population”. Scientometrics 104 (1): 1-18. https://doi.org/10.1007/s11192-015-1593-7

Ortega, José Luis. 2015b. "Disciplinary differences in the use of academic social networking sites". Online Information Review 39 (4): 520-536. https://doi.org/10.1108/OIR-03-2015-0093

Ovadia, Steven. 2013. "When Social Media Meets Scholarly Publishing". Behavioral ESocial Sciences Librarian 32, 194-198. https://doi.org/10.1080/01639269.2013.817886

Prieto Gutiérrez, Juan José. 2018. "Presencia de autores en ResearchGate y Google Scholar. El caso del Área de Biblioteconomía y Documentación (LIS)”, in Conocimientos sin fronteras: colaboración científica e institucional en documentación e información, 85-98. Madrid: Universidad Complutense https://dialnet.unirioja.es/servlet/extart?codigo=7015905

Rodríguez Bravo, Blanca y David Nicholas. 2020. "Descubrir, leer, publicar, compartir y monitorizar el progreso: comportamiento de los investigadores junior españoles". Elprofesional de la información 29 (5): e290503. https://doi.org/10.3145/epi.2020.sep.03

Ruiz-Pérez, Sergio y Emilio Delgado-López-Cózar. 2017. "Opiniones, actitudes y prácticas de los investigadores españoles hacia la publicación en acceso abierto”. Elprofesional de la información 26 (4): 722-734.

https://doi.org/10.3145/epi.2017.jul.16

Sánchez Pérez, Javier. 2020. "Hacia la colaboración entre repositorios y redes sociales académicas”. Revista PH 100: 120-121. https://doi.org/10.33349/2020.100.4666

Scherer, David. 2016. "Incentivizing Them to Come: Strategies, Tools, and Opportunities for Marketing an Institutional Repository”, en Making Institutional Repositories Work, 159-174. EUA: Purdue University Press. https://doi.org/10.2307/j.ctt1wf4drg.17 
Thelwall, Mike y Kayvan Kousha. 2015. "ResearchGate: Disseminating, Communicating, and Measuring Scholarship?" Journal of the Association for Information Science and Technology 66 (5): 876-889. https://doi.org/10.1002/asi.23236

Xia, Feng, Xiaoyan Su, Wei Wang, Chenxin Zhang, Zhaolong Ning e Ivan Lee. 2016. "Bibliographic Analysis of Nature Based on Twitter and Facebook Altmetrics Data". PLoS ONE 11 (12): e0165997. https://doi.org/10.1371/journal.pone.0165997

Yan, Weiwei, Yin Zhang, Tao Hu y Sonali Kudva. 2021. "How Does Scholarly Use of Academic Social Networking Sites Differ by Academic Discipline? A Case Study Using ResearchGate”. Information Processing and Management 58 (1): 102430. https://doi.org/10.1016/j.ipm.2020.102430

Para citar este texto:

Fernández-Ramos, Andrés y Leticia Barrionuevo. 2022. "La difusión de la producción científica en el ámbito de las Humanidades: el caso de la Universidad de León”. Investigación Bibliotecológica: archivonomía, bibliotecología e información 36 (90): 47-65.

http://dx.doi.org/10.22201/iibi.24488321xe.2022.90.58486 
Anexo. Distribución de los investigadores en cada área temática y departamento

\begin{tabular}{|c|c|}
\hline Departamentos / Áreas de conocimiento & Investigadores \\
\hline Filología Hispánica y Clásica & 26 \\
\hline Didáctica de la lengua y la literatura & 3 \\
\hline Filología griega & 1 \\
\hline Filología latina & 2 \\
\hline Lengua española & 5 \\
\hline Lingüística general & 7 \\
\hline Literatura española & 6 \\
\hline Teoría de la literatura y literatura comparada & 2 \\
\hline Filología Moderna & 24 \\
\hline Filología francesa & 4 \\
\hline Filología inglesa & 20 \\
\hline Geografía y Geología & 12 \\
\hline Análisis geográfico regional & 3 \\
\hline Geografía física & 4 \\
\hline Geografía humana & 5 \\
\hline Historia & 21 \\
\hline Antropología social & 2 \\
\hline Historia antigua & 2 \\
\hline Historia contemporánea & 4 \\
\hline Historia de América & 1 \\
\hline Historia medieval & 4 \\
\hline Historia moderna & 4 \\
\hline Prehistoria & 4 \\
\hline Patrimonio Artístico y Documental & 13 \\
\hline Ciencias y técnicas historiográficas & 3 \\
\hline Historia del arte & 10 \\
\hline Total & 96 \\
\hline
\end{tabular}

